\title{
Myalgic encephalomyelitis / chronic fatigue syndrome as a breakdown of homeostasis
}

David F Marks

Funding: The author(s) received no specific funding for this work.

Potential competing interests: The author(s) declared that no potential competing interests exist.

\section{Abstract}

Homeostasis is a fundamental physiological principle that ensures equilibrium, stability and safety of the organism in a continuously changing and potentially lifethreatening environment. This article introduces a new theory of the aetiology of ME/CFS that hypothesises that ME/CFS is caused by a breakdown of homeostasis that produces an aberrant state of disequilibrium with endocrinological and immunological changes. The current hypothesis is that ME/CFS involves a changed set point such that homeostasis persistently pushes the organism towards a pathological dysfunctional state because the disequilibrium fails to reset. To use an analogy of a thermostat, if the 'off switch' of a thermostat stops working, the house would become warmer and warmer without limit. Here I summarise the evidence in support of the theory. Clinical trials to investigate the role of suggested biological mediators such as thyrotropin-releasing hormone in ME/CFS would appear to be helpful.

\section{Abbreviations}

ANS, autonomic nervous system; ATP, adenosine triphosphate; CRF, cancer-related fatigue; CNS, central nervous system; EAS, extended autonomic system; HPA, hypothalamic-pituitary-adrenal; ME/CFS, myalgic encephalomyelitis/chronic fatigue syndrome; MUS, medically unexplained symptoms; SNS, sympathetic nervous system; RAG, recombination-activating gene; TAL, taltirelin; TRH, thyrotropin-releasing hormone.

Introduction

ME/CFS is a serious, unpredictable, complex, multisystem, chronic disease that often profoundly limits the health and activities of affected patients. It affects approximately 30 million people worldwide. The Institute of Medicine's (IOM; 2015) diagnostic criteria for ME/CFS are shown in Box 1: 


\section{BOX 1 \\ Proposed Diagnostic Criteria for ME/CFS}

Diagnosis requires that the patient have the following three symptoms:

1. A substantial reduction or impairment in the ability to engage in preillness levels of occupational, educational, social, or personal activities, that persists for more than 6 months and is accompanied by fatigue, which is often profound, is of new or definite onset (not lifelong), is not the result of ongoing excessive exertion, and is not substantially alleviated by rest, and

2. Post-exertional malaise, ${ }^{*}$ and

3. Unrefreshing sleep*

At least one of the two following manifestations is also required:

1. Cognitive impairment* or

2. Orthostatic intolerance

* Frequency and severity of symptoms should be assessed. The diagnosis of ME/CFS should be questioned if patients do not have these symptoms at least half of the time with moderate, substantial, or severe intensity.

For more information, visit www.iom.edu/MECFS

(20) INSTITUTE OF MEDICINE

Advising the nation - Improving health

It is informative to mention other ME/CFS manifestations listed by the IOM (2015):

- Pain - very common, but highly variable in presence, nature and severity.

- Certain infections may act as triggers

- Gastrointestinal and genitourinary problems

- Sore throat or scratchy throat

- Painful or tender axillary/cervical lymph nodes

- Sensitivity to external stimuli

Substantial decrease or impairment in function with profound fatigue reported by the IOM included patient descriptions such as the following:

- "flu-like fatigue/ exhaustion"

- "I feel like a battery that is never able to be recharged fully despite resting a lot and limiting my activities to only the bare essentials needed to get by"

- "Thinking takes a lot more work than it used to"

- "My arms, legs, body feel heavy and harder to move"

- severe limitations in personal and household management

- loss of job, medical insurance and career

- being predominantly housebound

- decreased social interaction and increased isolation

Of key importance, is 'post exertional malaise' (PEM) which is a prolonged exacerbation of a patient's baseline symptoms after physical/ cognitive/orthostatic exertion or stress. It may be delayed relative to the trigger. Patient descriptions include: "crash," "relapse," "collapse", being mentally tired after the slightest effort, being physically drained or sick after mild activity, the more demanding, prolonged or repeated the activity, the more severe and prolonged the payback. Other important symptoms include: Unrefreshing sleep: Feeling unrefreshed after sleeping many hours. Cognitive impairments: Problems with thinking exacerbated by exertion, effort, 
or stress or time pressure. Orthostatic intolerance: Symptoms worsen upon assuming and maintaining upright posture and are improved, though not necessarily abolished, by lying back down or elevating feet (IOM, 2015).

There is no agreed scientific explanation of the disorder(s), no diagnostic marker, no treatment and no cure. Although the cause of ME/CFS is unknown, in many cases, symptoms may have been triggered by an infection or other prodromal event, such as "immunization, anaesthetics, physical trauma, exposure to environmental pollutants, chemicals and heavy metals, and rarely blood transfusions" (IOM, 2015; Carruthers and van de Sande, 2005, p. 1).

An unconfirmed psychiatric theory has been that ME/CFS is a post-viral psychosomatic condition based on dysfunctional beliefs, deconditioning and biased attention (Wessely, David, Butler \& Chalder, 1989). A recent review suggests that none of the hypotheses are supported by empirical evidence and, furthermore, treatments using the psychosomatic theory have caused patient harms (Marks, 2021). The psychosomatic theory neglects the burgeoning literature on the biological mechanisms of MUS/ME/CFS, the topic of the current paper.

Here, I discuss a biological explanation of these complex and unpredictable conditions as a breakdown of the body's protective system of homeostasis. All physical and mental disorders are caused by temporary homeostatic changes. It might therefore be said that there is nothing particularly novel about the current hypothesis. It has also been previously suggested that aberrant homeostasis may play a role in the aetiology of ME/CFS, e.g. Arroll (2013), Hatziagelaki et al. (2018), Nacul et al. (2020), so what is new here? The current theory is unique in proposing that, unlike illnesses that typically show recovery when equilibrium returns to a previously established or 'normal' set point, ME/CFS involves a changed set point such that homeostasis persistently pushes the organism towards a pathological dysfunctional state so that the disequilibrium fails to reset. The hypothesis is illustrated in Figure 1. 

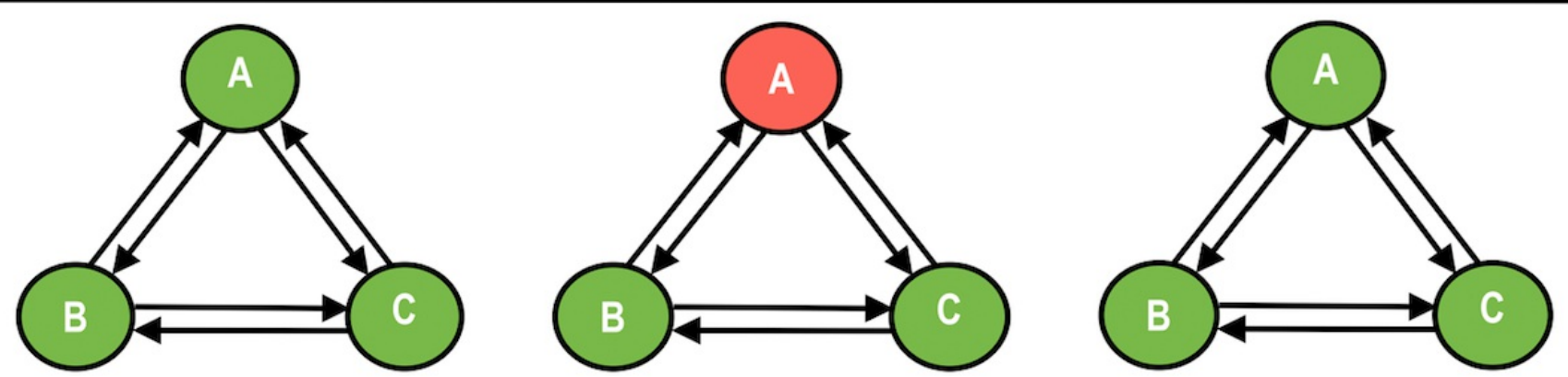

\section{Homeostasis with reset function}
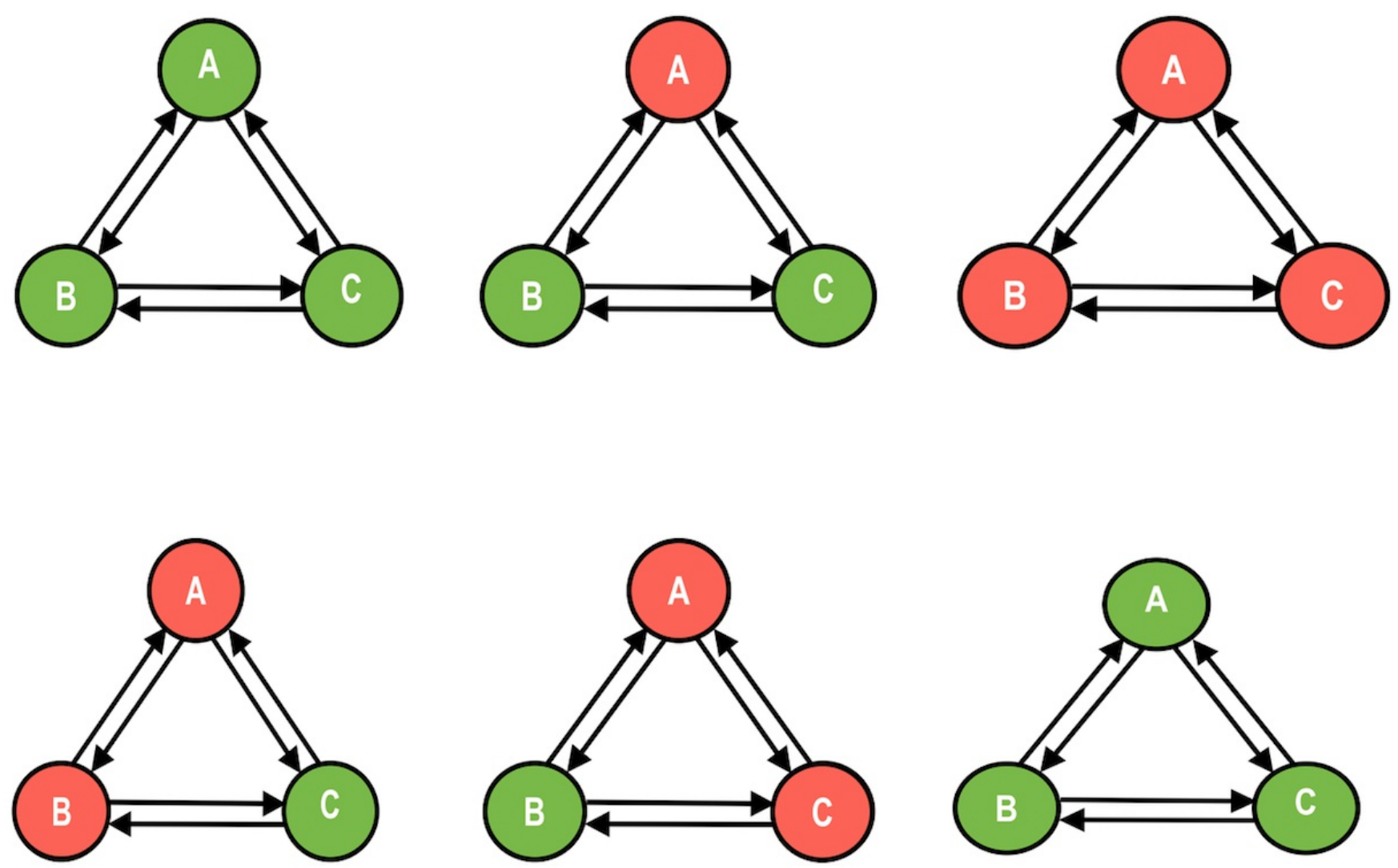

\section{Homeostasis without reset function}

Figure 1. Three processes, A, B and C, exist in homeostasis, which normally resets equilibrium following any disruption. The upper panel illustrates the normally functioning case of homeostasis with reset: on the left, $\mathrm{A}, \mathrm{B}$ and $\mathrm{C}$ are in equilibrium (green). In the centre, A has fallen out of equilibrium (red) and requires a reset. On the right, $\mathrm{A}$ is reset and the three processes are returned to equilibrium. The lower panel shows an aberrant, non-resetting homeostasis as hypothesised in the aetiology of ME/CFS: in the upper row, left, the system shows $A, B$ and $C$ in equilibrium. In the centre, A is out of equilibrium and requires a reset. On the right, the system fails to reset and remains in disequilibrium. In the lower row, the system remains unstable: on the left, $A$ and $B$ both are out of equilibrium, in the centre, $\mathrm{A}$ and $\mathrm{C}$ are out of equilibrium. Finally, the system is reset to equilibrium.

One of many scientific mysteries about ME/CFS has been its highly fluctuating symptom profile with a predominant tendency to wax and wanefriedman, Bateman, Bested, and Nahle, 2019; Morris, Anderson, Galecki, Berk and Maes, 2013; Morris and Maes, 2014). Schei and Angelsen (2021) report a patient survey with 5,822 ME patients in Norway. In regard to the course of the illness, the authors state: "Large fluctuations, or fluctuations with gradual deterioration, are the two most typical courses of the illness". The non-resetting homeostasis theory proposed here is consistent with the unpredictability of ME/CFS symptoms and the waxing and waning tendency showing widely fluctuating symptoms from time to time. In the next sections, I briefly introduce the body's main systems for homeostasis and review the evidence on the connections between disruptions of bodily homeostasis and the illnesses known as 'ME/CFS'. 
Physiological Homeostasis

The body uses three biological systems for the maintenance of equilibrium: the autonomic nervous system, the endocrine system and the immune system. The three systems communicate using electrical and chemical signals coordinated by the brain. They activate and de-activate tissues and organs to control and regulate the body, the emotions and behaviour. The principal objective of the three systems is to preserve homeostasis. It is proposed that the disorder known as ME/CFS is caused by a breakdown of homeostasis. A 'Homeostasis Breakdown Theory of ME/CFS' is supported by a number of studies in the recent literature. The three systems of homeostasis and their relationships to the brain and behaviour are illustrated in Figure 2.

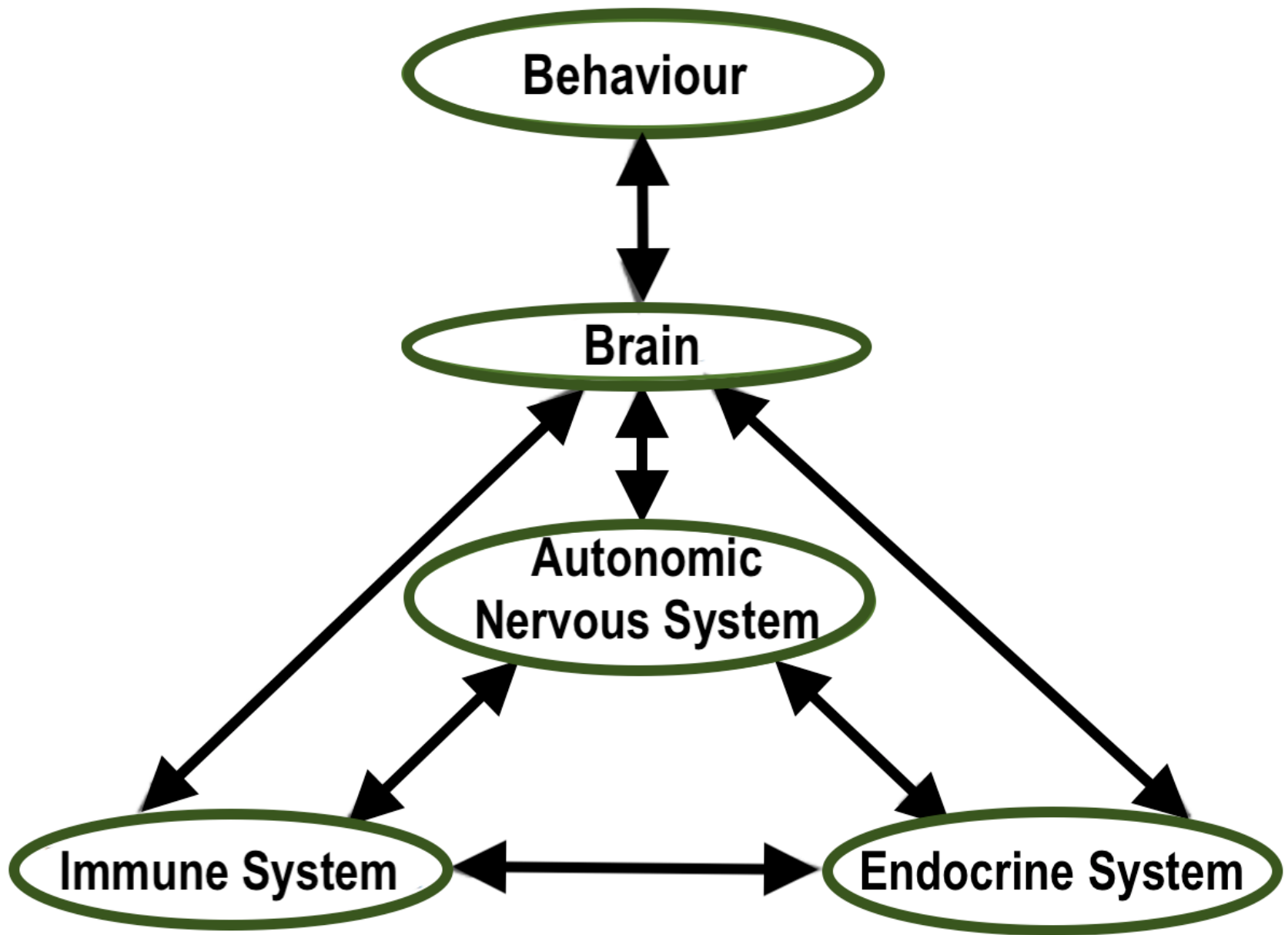

Figure 2. The systems of homeostasis provides by the autonomic, endocrine and immune systems, and their relationships to the brain and behaviour.

Continuous, reciprocal interaction between the nervous system, organs and gut is essential for smooth and efficient control and coordination of bodily functions, experience and behaviour. Endocrine substances directly affect the nervous and immune systems. The CNS innervates every organ and tissue of the immune system with reciprocal connections. The continuous interactions of the nervous, endocrine and immune systems was termed 'neuroimmunomodulation' by Spector and Korneva (1981). The neuroendocrine and neuroimmune systems are so well integrated with the ANS that they act as parts of an "extended autonomic system" (EAS) A hierarchical brain network-the "central autonomic network"-regulates these systems (Goldstein 2020). Acute, coordinated alterations in homeostatic settings are believed to be crucial for surviving stressors. However, it is thought that intense or long-term EAS activation may cause harm because "EAS activation in the setting of chronically decreased homeostatic efficiencies (dyshomeostasis) may reduce thresholds for induction of destabilizing, lethal vicious cycles" (Goldstein, 2020).

If a breakdown in homeostasis occurs, and no reset is possible, an individual is in serious trouble. This may precisely be the case in people with ME/CFS (pwME/CFS) and with other forms of MUS. The chronic, fluctuating nature of ME/CFS suggests that its pathogenesis involves a disruption of homeostasis leading to chronic inflammation and immunological dysfunction. Biomarkers of inflammation and leaky gut syndrome as a possible result of microbiome disturbance and bacterial

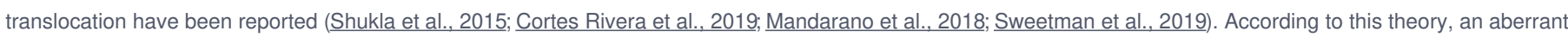
state of homeostasis is the central cause of ME/CFS ( $\underline{\text { Hatziagelaki et al., 2018; }}$ Nacul et al., 2020; Sweetman et al., 2019; Sweetman et al., 2020)

Stress is known to suppress immune function and increase susceptibility to infections and cancer (Dahbhar, 2009). Antoni and Weiss (2003) reviewed evidence on the role of stress and immunity in people with chronic fatigue syndrome. The immunosuppressive effects of increased cortisol on some specific immune cells, such as neutrophils and natural killer cells have been reported (e.g. Tønnesen, Christensen \& Brinkløv, 1987). Increased stress, as well as viral infections, will increase cortisol, 
possibly leading to immunosuppression and contributing to the development of ME/CFS. In line with what is known about the typical course of the disease, it can reasonably be assumed that the hypothesized state of aberrant homeostasis can be aggravated by new stressors in the form of infection (Shepherd \& Chaudhuri, 2019), physical exertion, cognitive effort such as reading or solving mental puzzles, triggering a post-exertional malaise (PEM), comorbid conditions such as sleep disturbances (Chu, Valencia, Garvert \& Montoya, 2018) and other factors Nacul et al.. 2020). In people who happily do not develop ME/CFS or prolonged illness following an acute infection or other insult, external stressors initially cause short-term physiological changes but the usual state of homeostatic equilibrium that operated before the insult is quickly restored.

Hatziagelaki et al. (2018) suggested that the aberrant state of homeostasis could be caused by inflammation in the hypothalamus. However, it must be noted that the aberrant state of homeostasis proposed by Hatziagelaki et al. (2018) to result from hypothalamic inflammation is unconfirmed. No such inflammation has ever been found in microscopic slices or other techniques and homeostatic disequilibrium can occur through disturbed regulatory systems without any focal inflammation in the brain. Here I consider four hypotheses for the presentation of the symptoms and their persistence in patients with ME/CFS: the neuroimmune hypothesis, the microbiome hypothesis, the mitochondrial failure hypothesis and the thyrotropin-releasing hormone hypothesis.

\section{The Neuroimmune Hypothesis}

Patients with ME/CFS often present with an acute onset of illness, with symptoms similar to an influenza infection, but these symptoms do not subside. Many of the symptoms are inflammatory, i.e. myalgia, arthralgia or joint pains, sore throat and tender lymphadenopathy (Maes, Twisk, Kubera \& Ringel, 2012). The immune system is a network of cells, tissues and organs that protects the body against disease or other potentially damaging foreign bodies. When properly functioning, the system identifies and attacks a variety of threats using billions of diverse antibodies, including viruses, bacteria and parasites, while distinguishing them from the body's own

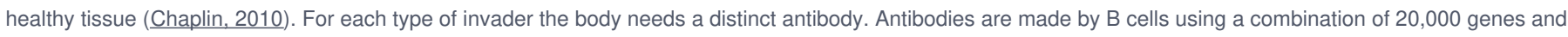
enzymes called 'RAG1' and 'RAG2' which are DNA shufflers (Khan \& Ali, 2017). These processes enables the immune system to create a vast diversity of antibodies and respond to diseases it has never encountered before.

Inflammation is another critical defence response in our innate immune system wherein white blood cells protect us from infection by foreign organisms, bacteria and viruses (Muralidharan \& Mandrekar, 2013). Inflammation occurs following infection or tissue damage when a rapid and complex series of reactions takes place to prevent tissue damage, isolate and destroy the infective organism, conserve and protect some micronutrients and activate the repair processes to restore normal functioning (Nathan, 2002). Inflammation is a homeostatic process that is only intended to last a few days but, if it is continued indefinitely, there is a poor prognosis in many conditions. Inflammatory responses take precedence over normal body metabolism with the objective of restoring normality as quickly as possible.

Immune activation indicated by elevated levels of cytokines in pwME/CFS have been studied since 2001 Patarca, 2001). Recent studies have used immune system profiling to determine whether an abnormal profile of circulating cytokines could be identified in pwME/CFS and whether this profile correlated with disease severity and/or fatigue duration. In one study, serum cytokines of 192 pwME/CFS and 392 healthy controls were measured (Montoya et al., 2017). Seventeen cytokines showed a statistically significant upward linear trend that correlated with ME/CFS severity of which 13 were proinflammatory, which is likely to be a contributing factor to the symptoms.

Strawbridge, Sartor, Scott and Cleare (2019) carried out a systematic review and meta-analysis of 42 studies of circulating inflammatory proteins in pwCFS and nonaffected control groups. Patients with CFS had significantly elevated tumour necrosis factor, interleukin-2, interleukin-4, transforming growth factor- $\beta$ and c-reactive protein. The authors concluded: "These data provide some support for an inflammatory component in CFS, although inconsistency of results indicates that inflammation is unlikely to be a primary feature in all those suffering from this disorder."

The generic cause of the abnormal inflammatory response in pwME/CFS appears to be a breakdown of some kind in homoeostasis. However, the precise mechanism remains uncertain (Komaroff, 2019). A review by Mensah et al. (2017) concluded that consistency in cytokine data is lacking and it is impossible to attribute their role as causal of the disease or its course.

\section{The Microbiome Hypothesis}

The field of microbiome research studies the microbes within the gut and the effects of these microbes on the host's well-being. The microbiome is the community of symbiotic and pathogenic microorganisms, that live on the skin and genitals and in the nose, ears, mouth and gut. Microbes influence metabolism, immunity and behaviour (Wong et al., 2015). One mechanism appears to involve hormones because specific changes in hormone levels correlate with the presence of the gut microbiota (Neuman et al., 2015). The microbiota produce and secrete hormones, respond to host hormones and regulate expression levels of host hormones. Hormones and the microbiome are linked to immune responses under both healthy conditions and autoimmune disease (Furness, Kunze \& Clerc, 1999). There are many interconnections and the microbiome and hormones may work through shared pathways to affect the immune response (Neuman et al., 2015). 
Organisms within the gut play a role in the early programming and response to stress. The gut contains $10^{3}-10^{14}$ micro-organisms, ten times the number of cells in the human body, and contains $150-360$ times as many genes as our genome. When a pathogen such as Escherichia coli (E coli) enters the gut, the HPA axis can be activated (Dinan \& Cryan, 2012). Stress can induce increased permeability of the gut, allowing bacteria and bacterial antigens to cross the epithelial barrier and to activate an immune response, which alters the content of the microbiome and lead to further increases in the HPA axis drive (Sudo, 2014).

Patients with irritable bowel syndrome and other MUS show alterations of the HPA axis which are induced by increased gut permeability. In the case of irritable bowel syndrome, the increased permeability can respond to probiotic therapy. The gut microbiota play a role in regulating the HPA axis. Verdino (2017) cautiously concludes his review of the connection between gut health and emotional well-being suggesting that nutritional interventions are unlikely to be a "panacea for profound psychological difficulties" (Verdino, 2017: 4).

The immune and neuroendocrine systems share a set of hormones and receptors. Glucocorticoids such as corticosterone and cortisol, regulate inflammation levels and have effects both on the innate and adaptive immune responses. Additionally, vitamin D affects immune cell responses by enhancing antigen presentation. Moreover, sex hormones affect the immune response in numerous ways.

Dysbiosis, an imbalance in the microbiome, seems to be involved in the pathophysiology of ME/CFS.Shukla et al., (2015) studied changes in the gut and plasma microbiome following exercise challenge in pwME/CFS. In exercise challenge, changes occurred in the amount of bacterial phyla in the gut in pwME/CFS that were not observed in healthy controls. Also, bacteria clearance from the blood was delayed in pwME/CFS following exercise. Altered gut microbiome and increased bacterial translocation following exercise thus appear to play a role in ME/CFS that may account for the post-exertional malaise experienced by many pwME/CFS. The authors concluded that their results provide evidence for a systemic effect of an altered gut microbiome in ME/CFS patients compared to controls. Nagy-Szakal et al. (2017) partially confirmed the findings of Shukla et al. (2015) in being able to differentiate the faecal metagenomic profiles of patients with ME/CFS from those of people with irritable bowel syndrome.

\section{Mitochondrial Failure}

Mitochondial failure is another part of the jigsaw puzzle posed by ME/CFS (Myhill et al. 2009). Mitochondria generate cellular energy by manufacturing ATP (adenosine triphosphate). Infections with pathogens, including viruses, bacteria, parasites and toxins can cause changes in the function of mitochondria and deplete energy metabolism. Metabolomics, the systematic identification of metabolic products (the metabolome) is indicating a possible chemical pattern of metabolite abnormalities in pwME/CFS. Myhill et al. (2009) developed interventions based on the illness' biochemistry, specifically the role of mitochondria in producing ATP, the energy currency used for body functions including the recycling of ADP to replenish the supply of ATP. Patients with ME/CFS, diagnosed using the Centers for Disease Control criteria, yielded a correlation between the degree of mitochondrial dysfunction and the severity of illness $(P<0.001)$ with only 1 of 71 patients overlapping with the normal region. The authors concluded that the 'ATP profile' can differentiate patients who have fatigue and other symptoms as a result of energy wastage by stress and psychological factors from those who have insufficient energy due to cellular respiration dysfunction (Myhill et al., 2009).

Morris and Maes (2014) supported Myhill et al.'s approach in arguing that mitochondrial dysfunctions in the form of lowered ATP production may play a role in the onset of ME/CFS symptoms and help to explain in part the central metabolic abnormalities observed in ME/CFS, e.g. glucose hypometabolism and cerebral hypoperfusion. The findings of Sweetman et al., (2020) also support a model of deficient ATP production in people with ME/CFS.

\section{The Thyrotropin-Releasing Hormone (TRH) Hypothesis}

Hypoactivity of the HPA axis appears likely to have a mediating role in the aetiology of ME/CFS as a consequence of activated immune-inflammatory and oxidative and nitrosative pathways according to Morris, Anderson and Maes (2017). Thyrotropin-releasing hormone (TRH), also termed thyroliberin, serves as a regulator of the HPA axis (Jackson, 1982) but its role likely extends beyond this. The body's TRH is mainly produced by nuclei of the hypothalamus, the key regulator of arousal, sleep and wakefulness, metabolism, energy level, food intake, temperature, fluid, endocrine and reproductive functions, emotion, stress circadian rhythm, visceral function, reward and punishment. It would not be surprising if a hormone called 'thyrotropin-releasing hormone' (TRH) closely connected with hypothalamic functioning would be implicated in the aetiology of the complex, multisystemic condition of ME/CFS. A research group at the Connecticut School of Medicine, Department of Psychiatry, developed the "Hypothesis of Homeostatic Regulation" (Gary, Sevarino, Yarbrough, Prange \& Winokur, 2003), later expanded to the "TRH-immune system homeostatic hypothesis" by Jayesh Kamath and others (Kamath, Yarbrough, Prange \& Winokur, 2009).

The Connecticut group proposes that the main neurobiological function of TRH is to promote homeostasis, through mechanisms within four integrated systems: 1) the hypothalamic-hypophysiotropic neuroendocrine system, 2) the brainstem/midbrain/spinal cord system, 3) the limbic/cortical system, and 4) the chronobiological system. The group also refers to TRH's effect on the reversal of sedation in the hibernating ground squirrel (Stanton, Caine \& Winokur, 1992). The evidence indicates that $\mathrm{TRH}$ is a homeostatic agent that opposes many perturbations in the CNS and ANS which tends to restore functioning to normal limits. From an evolutionary 
perspective, Kamath et al. suggested that, , there would be an advantage in having a direct pathway for suppression of TRH (Figure 3) which may be necessary for survival during an adaptive sickness response associated with proinflammatory cytokines acting in the brain (Kelley et al., 2003).

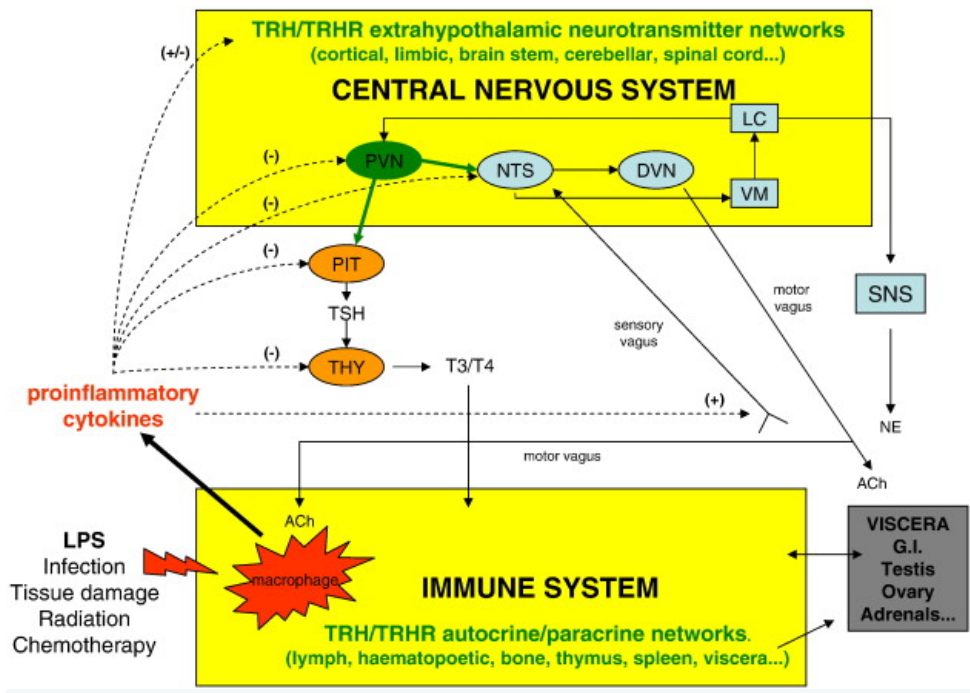

Figure 3. The thyrotropin-releasing hormone (TRH)-immune system homeostatic hypothesis. Reproduced from Kamath et al. (2009) by permission.

Kamath et el. (2009) trialed TRH for the treatment of cancer-related fatigue. Despite the half-life of TRH being only 5 minutes, they found that a single infusion of TRH led to significant and long-lasting improvements in energy levels and improved quality-of-life indicators. It is uncertain whether these effects are central, peripheral or a combination of the two. A similar homeostatic mechanism could underlie the fatigue and other complex symptoms of ME/CFS.

Recent studies have focused on the similarities between the fatigue in cancer patients and patients with ME/CFS. Cancer-related fatigue (CRF) is not relieved by rest, can decrease quality of life, and there is no FDA-approved therapy. Dougherty et al. (2017) tested the TRH analogue, taltirelin (TAL), in mouse models of CRF. They used a mouse model of chemotherapy, a mouse model of radiation therapy, and mice bearing colon 26 carcinoma tumours and a treadmill fatigue test to assess fatigue-like behaviour after treatment with TAL. Additionally, wild-type and TRH receptor knockout mice were used to determine which TRH receptor was necessary for the actions of TAL. Tumour-bearing mice displayed muscle wasting and all of the models caused fatigue-like behaviour, producing shorter running distances than the control mice. The authors concluded that TAL reversed fatigue-like behaviour in all three models and that the mouse TRH1 receptor was necessary for the effects of TAL. The findings of Dougherty et al. suggest that TAL may be helpful in alleviating fatigue in cancer patients and possibly ME/CFS patients also. Therapeutic use of $\mathrm{TRH}$ is presently restricted to spinocerebellar degenerative disease. However, pending necessary approvals, a controlled evaluation of TAL as a potential therapy for ME/CFS in humans appears warranted.

\section{Conclusion}

A breakdown of the reset function of homeostasis provides a plausible theory to explain the aetiology and fluctuating symptoms of ME/CFS. The hypothesis receives tentative support from a number of domains including the evidence of mitochondrial dysfunction that lowers ATP production and the evidence regarding TRH and TAL suggesting the possibility that these may be helpful in alleviating fatigue in patients with cancer and possibly ME/CFS also. If possible, randomised controlled trials of $\mathrm{TRH} / \mathrm{TAL}$ with patients in the advanced stages of ME/CFS would be a useful forward step. The current theoretical approach is speculative and requires in-depth investigation before any definite conclusions can be drawn.

\section{Statements and Declarations}

Data Sharing Statement

The research for this article did not include the collection of new data.

Ethical Statement

No new data were collected with human or non-human participants.

Conflict of Interests 
The author has no conflicts of interest to declare.

Funding

The author received no funding for the research reviewed in this article.

Acknowledgements

The author thanks three reviewers for their helpful comments on an earlier version of this manuscript: Suzanne Broadbent, Ewa Klaver-Krol, and Tse-Yen Yang. Dominic Stanculescu contributed input to the section on the TRH hypothesis, which will be expanded in a further publication (Marks \& Stanculescu, 2021).

\section{References}

- Antoni MH and Weiss DE. (2003). Stress, immunity and chronic fatigue syndrome: A conceptual model to guide the development of treatment and research. In: Jason PF LA, Taylor R, editors. Handbook of chronic fatigue syndrome and fatiguing illnesses. New York: John Wiley \& Sons, Inc.

- Arroll, M. A. (2013). Allostatic overload in myalgic encephalomyelitis/chronic fatigue syndrome (ME/CFS) Medical hypotheses, 81(3), 506-508.

- Carruthers, B. M., and M. I. van de Sande (2005). Myalgic encephalomyelitis/chronic fatigue syndrome. A clinical case definition and guidelines for medical practitioners. An overview of the Canadian consensus document. Vancouver, BC: Carruthers and van de Sande.

- Centers for Disease Control and Prevention (2021). https://www.cdc.gov/mecfs/index.htm|\#: :text=Myalgic\%20encephalomyelitis\%2Fchronic\%20fatigue\%20syndrome\%20(ME\%2FCFS)\%20is,severe\%20fatigue\%20and\%20sleep\%20problems.

- Chaplin, D. D. (2010). Overview of the immune response. Journal of Allergy and Clinical Immunology, 125(2), S3-S23.

- Chu, L., Valencia, I. J., Garvert, D. W., \& Montoya, J. G. (2018).Deconstructing post-exertional malaise in myalgic encephalomyelitis/chronic fatigue syndrome: A patient-centered, cross-sectional survey. PloS one, 13(6), e0197811.

- Cortes Rivera, M., Mastronardi, C., Silva-Aldana, C. T., Arcos-Burgos, M., \& Lidbury, B. A. (2019).Myalgic encephalomyelitis/chronic fatigue syndrome: a comprehensive review. Diagnostics, 9(3), 91.

- Dhabhar, F. S. (2009). Enhancing versus Suppressive Effects of Stress on Immune Function: Implications for Immunoprotection and Immunopathology. Neuroimmunomodulation, 16(5), 300-317.

- Dinan, T. G., \& Cryan, J. F. (2012).Regulation of the stress response by the gut microbiota: implications for psychoneuroendocrinology Psychoneuroendocrinology, 37(9), 1369-1378.

- Dougherty, J. P., Wolff, B. S., Cullen, M. J., Saligan, L. N., \& Gershengorn, M. C. (2017).Taltirelin alleviates fatigue-like behavior in mouse models of cancer-related fatigue. Pharmacological research, 124, 1-8.

- Friedman, K. J., Bateman, L., Bested, A., and Nahle, Z. (2019).Advances in ME/CFS Research and Clinical Care. Frontiers in Pediatrics, 7, 370.

- Furness, J. B., Kunze, W. A., \& Clerc, N. (1999). II.The intestine as a sensory organ: neural, endocrine, and immune responses. American Journal of PhysiologyGastrointestinal and Liver Physiology, 277(5), G922-G928.

- Gary, K. A., Sevarino, K. A., Yarbrough, G. G., Prange, A. J., \& Winokur, A. (2003).The Thyrotropin-Releasing Hormone (TRH) Hypothesis of Homeostatic Regulation: Implications for TRH-Based Therapeutics. Journal of Pharmacology and Experimental Therapeutics 305(2), $410-416$.

- Goldstein, D. S. (2020). The extended autonomic system, dyshomeostasis, and COVID-19. Clinical Autonomic Research, 1-17.

- Hatziagelaki, E., Adamaki, M., Tsilioni, I., Dimitriadis, G., \& Theoharides, T. C. (2018).Myalgic encephalomyelitis/chronic fatigue syndrome-metabolic disease or disturbed homeostasis due to focal inflammation in the hypothalamus? Journal of Pharmacology and Experimental Therapeutics 367(1), $155-167$.

- Institute of Medicine (2015). Beyond Myalgic Encephalomyelitis/Chronic Fatigue Syndrome: Redefining an IIIness. Washington, DC: The National Academies Press. https://doi.org/10.17226/19012. Available at: https://www.nap.edu/catalog/19012/beyond-myalgic-encephalomyelitischronic-fatigue-syndrome-redefining-an-illness. Accessed on 29 May 2021.

- Jackson, I. M. (1982). Thyrotropin-releasing hormone. New England Journal of Medicine, 306(3), 145-155.

- Kamath, J., Yarbrough, G. G., Prange Jr, A. J., \& Winokur, A. (2009). The thyrotropin-releasing hormone (TRH)-immune system homeostatic hypothesis. Pharmacology \& therapeutics, 121(1), 20-28.

- Kelley, K. W., Bluthé, R. M., Dantzer, R., Zhou, J. H., Shen, W. H., Johnson, R. W., \& Broussard, S. R. (2003)ytokine-induced sickness behavior. Brain, behavior, and immunity, 17(1), 112-118.

- Khan, F. A., \& Ali, S. O. (2017). Physiological Roles of DNA Double-Strand Breaks. Journal of nucleic acids, 2017.

- Komaroff, A. L. (2019). Advances in understanding the pathophysiology of chronic fatigue syndrome JAMA, 322(6), $499-500$.

- Maes, M., Twisk, F. N., Kubera, M., \& Ringel, K. (2012).Evidence for inflammation and activation of cell-mediated immunity in myalgic encephalomyelitis/chronic fatigue syndrome (ME/CFS): increased interleukin-1, tumor necrosis factor- $\alpha$, PMN-elastase, lysozyme and neopterin. Journal of affective disorders, 136(3), 933- 
939.

- Mandarano, A. H., Giloteaux, L., Keller, B. A., Levine, S. M., \& Hanson, M. R. (2018).Eukaryotes in the gut microbiota in myalgic encephalomyelitis/chronic fatigue syndrome. PeerJ, 6, e4282.

- Marks, D.F. (2021). The rise and fall of the Wessely School.Health Psychology Open (under review).

- Marks, D.F., Murray, M. and Estacio, E.V. (2020) Health psychology. Theory, research \& practice. ( $\boldsymbol{f}^{\text {th }}$ ed). London: Sage Publications.

- Marks, D F \& Stanculescu, D. (2021). In preparation.

- Mensah, F. K. F., Bansal, A. S., Ford, B., \& Cambridge, G. (2017).Chronic fatigue syndrome and the immune system: where are we now? Neurophysiologie Clinique/Clinical Neurophysiology, 47(2), 131-138.

- Morris, G., Anderson, G., Galecki, P., Berk, M., and Maes, M. (2013).A narrative review on the similarities and dissimilarities between myalgic encephalomyelitis/chronic fatique syndrome (ME/CFS) and sickness behavior. BMC Medicine, 11(1), 1-19.

- Morris, G., \& Maes, M. (2014). Mitochondrial dysfunctions in myalgic encephalomyelitis/chronic fatigue syndrome explained by activated immuno-inflammatory. oxidative and nitrosative stress pathways. Metabolic brain disease, 29(1), 19-36.

- Morris, G., Anderson, G., \& Maes, M. (2017). Hypothalamic-Pituitary-Adrenal Hypofunction in Myalgic Encephalomyelitis (ME)/Chronic Fatigue Syndrome (CFS) as a Consequence of Activated Immune-Inflammatory and Oxidative and Nitrosative Pathways. Molecular neurobiology, 54(9), 6806-6819.

- Muralidharan, S., \& Mandrekar, P. (2013). Cellular stress response and innate immune signaling: integrating pathways in host defense and inflammation Journal of leukocyte biology, 94(6), 1167-1184.

- Myhill, S., Booth, N. E., \& McLaren-Howard, J. (2009). Chronic fatigue syndrome and mitochondrial dysfunction.International journal of clinical and experimental medicine, 2(1), 1.

- Nacul, L., O'Boyle, S., Palla, L., Nacul, F. E., Mudie, K., Kingdon, C. C., ... \& Lacerda, E. M. (2020) $\underline{\text { How myalgic encephalomyelitis/chronic fatigue syndrome }}$ (ME/CFS) progresses: The natural history of ME/CFS. Frontiers in neurology, 11, 826.

- Nagy-Szakal, D., Williams, B. L., Mishra, N., Che, X., Lee, B., Bateman, L., ... \& Lipkin, W. I. (2017) Fecal metagenomic profiles in subgroups of patients with myalgic encephalomyelitis/chronic fatigue syndrome. Microbiome, 5(1), 1-17.

- Nathan, C. (2002). Points of control in inflammation. Nature, 420(6917), 846-852.

- Neuman, H., Debelius, J. W., Knight, R., \& Koren, O. (2015).Microbial endocrinology: the interplay between the microbiota and the endocrine system FEMS microbiology reviews, 39(4), 509-521.

- Patarca, R. (2001). Cytokines and chronic fatigue syndrome. Annals of the New York Academy of Sciences 933(1), 185-200.

- Schei, T. and Angelsen, A. (2021). The course of the illness for ME patients in Norway: What are the typical courses of the illness, and what worsen or improve them? Norwegian ME Association. Available at:https://www.me-foreningen.no/wp-content/uploads/2021/03/Norwegian-ME-Association-2021-Report-on-the-courseof-illness-English-summary.pdf

- Shepherd, D. C., \& Chaudhuri, D. A. (2019).ME/CFS/PVFS: an exploration of the key clinical issues ME Association.

- Shukla, S. K., Cook, D., Meyer, J., Vernon, S. D., Le, T., Clevidence, D., ... \& Frank, D. N. (2015) challenge in myalgic encephalomyelitis/chronic fatigue syndrome (ME/CFS). PLoS One, 10(12), e0145453.

- Spector, N. H., \& Korneva, E. A. (1981). Neurophysiology, immunophysiology, and neuroimmunomodulation (Vol. 449). Academic Press, New York.

- Stanton, T. L., Caine, S. B., \& Winokur, A. (1992). Seasonal and state-dependent changes in brain TRH receptors in hibernating ground squirrels Brain research bulletin, 28(6), 877-886.

- Strawbridge, R., Sartor, M. L., Scott, F., \& Cleare, A. J. (2019).Inflammatory proteins are altered in chronic fatigue syndrome-a systematic review and metaanalysis. Neuroscience \& Biobehavioral Reviews, 107, 69-83.

- Sudo, N. (2014). Microbiome. HPA axis and production of endocrine hormones in the gut In Microbial endocrinology: the microbiota-gut-brain axis in health and disease (pp. 177-194). Springer, New York, NY.

- Sweetman, E., Noble, A., Edgar, C., Mackay, A., Helliwell, A., Vallings, R., ... \& Tate, W. (2019).Current research provides insight into the biological basis and diagnostic potential for myalgic encephalomyelitis/chronic fatigue syndrome (ME/CFS). Diagnostics, 9(3), 73.

- Sweetman, E., Kleffmann, T., Edgar, C., de Lange, M., Vallings, R., \& Tate, W. (2020)A SWATH-MS analysis of Myalgic Encephalomyelitis/Chronic Fatigue Syndrome peripheral blood mononuclear cell proteomes reveals mitochondrial dysfunction. Journal of translational medicine, 18(1), 1-18.

- Tønnesen, E., Christensen, N. J., \& Brinkløv, M. M. (1987). Natural killer cell activity during cortisol and adrenaline infusion in healthy volunteers European journal of clinical investigation, 17(6), 497-503.

- Verdino, J. (2017). The third tier in treatment: Attending to the growing connection between gut health and emotional well-being Health Psychology Open, 4(2), doi:10.1177/2055102917724335.

- Wessely, S., David, A., Butler, S., and Chalder, T. (1989). Management of chronic (post-viral) fatigue syndrome. The Journal of the Royal College of General Practitioners, 39(318), 26-29.

- Wong, A. C. N., Holmes, A., Ponton, F., Lihoreau, M., Wilson, K., Raubenheimer, D., \& Simpson, S. J. (2015) Behavioral microbiomics: a multi-dimensional 
approach to microbial influence on behavior. Frontiers in Microbiology, 6, 1359 